\title{
Raumverträglicher Ausbau von erneuerbaren Energien - ein alternativer Standortplanungsansatz für eine nachhaltige Energiewende
}

\author{
Stephan Bosch ${ }^{1}$, Joachim Rathmann ${ }^{2}$, and Florian Simetsreiter ${ }^{1}$ \\ ${ }^{1}$ Humangeographie, Institut für Geographie, Universität Augsburg, Augsburg, Germany \\ ${ }^{2}$ Physische Geographie, Institut für Geographie, Universität Augsburg, Augsburg, Germany \\ Correspondence to: Stephan Bosch (stephan.bosch@geo.uni-augsburg.de)
}

Received: 18 February 2015 - Revised: 21 January 2016 - Accepted: 26 January 2016 - Published: 23 February 2016

Kurzfassung. By now, there are numerous high-quality studies discussing the incompatibility of the development of renewable energies with the preservation of landscape. However, no further insight is provided as to how a holistic spatial integration of renewable energies may be achieved. Yet the ambitious aim of Germany's government of increasing the percentage of energy obtained from renewable energies to $45 \%$ by 2025 and to $60 \%$ by 2035 will lead to an even greater spatial strain on Germany's rural areas as it has been the case so far. Therefore, this study aims to critically investigate the currently established paradigm of site planning for renewable energies using GIS analysis. Based upon the findings, an alternative, holistic concept of site planning will be developed and exemplified.

\section{Standortsuche für erneuerbare Energien - theoretischer Hintergrund}

\subsection{Behavioristische Standortwahl}

Die Transformation der Energiewirtschaft - weg von fossilen und nuklearen Energieträgern, hin zu erneuerbaren Energien - geht mit erheblichen Veränderungen in der gesamtwirtschaftlichen Standortakquise von Kraftwerken zur Stromerzeugung einher. Dies ist dadurch zu erklären, dass im Zuge der Liberalisierung des Strommarktes die oligopolen Strukturen im Bereich der Energieversorgung sukzessive durch eine große Zahl an kleineren, weniger einflussreichen sowie semi-professionellen Akteuren aufgebrochen werden (Gailing und Röhring, 2015:34), die in anderer Art und Weise der Herausforderung der Standortsuche begegnen, als Großunternehmen dies tun.

Im Rahmen des noch dominierenden, jedoch rückzubauenden fossil-nuklearen Energiesystems werden Standortentscheidungen meist von spezialisierten Abteilungen großer Energieversorgungsunternehmen gefällt. Bathelt und Glückler (2002:133) verweisen darauf, dass „Entscheidungen großer Gremien z. B. bei der Standortwahl großer Unterneh- men einem entsprechend geringerem Fehlentscheidungsrisi$k o$ " unterliegen, als dies bei kleineren Unternehmungen der Fall ist. Erneuerbare-Energien-Anlagen sind i. d. R. kleinere Unternehmungen, oftmals in der Hand von Privatpersonen, Bürgerinitiativen und Energiegenossenschaften (Klagge und Brocke, 2013), deren Standortverhalten mitunter stark vom „mikrosozialen Umfeld“ (Klandt, 1984:59) geprägt wird. Der oft beschworene Vorteil einer demokratischeren Energieversorgung (Kunze und Becker, 2015) bringt folglich den Nachteil einer Entprofessionalisierung von Standortentscheidungsprozessen mit sich, deren geringe Qualität v. a. aus einem Defizit im Bereich der Informationsverarbeitung resultiert.

Von wesentlicher Bedeutung sind hierbei die Erkenntnisse aus der behavioristischen Standortlehre (Pred, 1967), die die Qualität einer Standortentscheidung in Abhängigkeit zur Verfügbarkeit sowie zur Verarbeitungsfähigkeit standortrelevanter Informationen seitens des Entscheidungsträgers sehen und suboptimalem Standortverhalten dadurch eine sehr hohe Wahrscheinlichkeit einräumen. Dies muss volkswirtschaftlich nicht zwangsläufig bedenklich sein, denn Smith (1971) verweist anhand des Marginalprinzips darauf, dass Unternehmer eben nicht zu einer punktbezogenen, sondern zu ei- 
ner flächenbezogenen Standortbewertung tendieren und eine Standortwahl jenseits des Standortoptimums vertretbar ist, wenn diese sich innerhalb der räumlichen Gewinnzone - der sog. margin - vollzieht. Die hieraus resultierende Wahlfreiheit birgt dennoch die Gefahr, dass in Abhängigkeit vom Unternehmertyp Standorte außerhalb der „,margins“ gewählt werden und in der Summe dieser einzelwirtschaftlichen Fehlentscheidungen eine gesamtwirtschaftliche Krise erwächst.

Da es sich im Rahmen eines regenerativen Energiesystems um viele zehntausende, dezentral orientierte sowie lokal getroffene Standortentscheidungen handelt - Ende $2015 \mathrm{gab}$ es 25980 Standorte von Windkraftanlagen in Deutschland -, die noch dazu stark in die Alltagsräume der Menschen eingreifen (Schöbel, 2012), erscheint es angebracht, Möglichkeiten der Optimierung von Standortentscheidungsprozessen zu erneuerbaren Energien auszuloten und zur Diskussion zu stellen. Die Einflussgrößen auf das unternehmerische Standortverhalten, die aus den verhaltenswissenschaftlichen Ansätzen bekannt sind, - bspw. stark eingegrenzte Standortsuchräume (Ballestrem, 1974), Limitierungen hinsichtlich Zeit, Geld und Information (Schmude, 1994:78), subjektive Wertvorstellungen und persönliche Präferenzen (Schätzl, 2001:60,63) sowie Nachahmungseffekte und Zufall (Kulke, 2013:99) - erreichen im Zuge der Energiewende eine Dimension, die über deren Gelingen entscheiden kann.

\subsection{Aufwertung ökologischer und sozialer Faktoren}

Die räumlichen Kosten- und Erlösfunktionen der Standortentscheidungen lassen sich dabei jedoch nicht allein auf die unternehmerische und damit vorwiegend ökonomische Perspektive reduzieren, vielmehr speist sich die teils vehemente Kritik am Ausbau erneuerbarer Energien aus ökologischen und sozialen Verwerfungen (Weiss, 2013; Laggner et al., 2014), die möglicherweise das Ergebnis wenig differenzierter Standortentscheidungsprozesse sind.

Die Überbewertung ökonomischer (Standort-)Faktoren bzw. die Unterbewertung ökologischer und sozialer Aspekte - vgl. Czisch (2005) sowie Prinz et al. (2009) - resultiert nach Daly (2001) nicht zuletzt aus der weit verbreiteten Vorstellung, dass das Ökosystem ein Subsystem der Wirtschaft und seine Zerstörung dadurch zu kompensieren sei, indem Natur-Kapital durch menschengemachtes Kapital substituiert wird. Im Kontext der ökologischen Ökonomik, in der umgekehrt die Wirtschaft das Subsystem des räumlich begrenzten, nicht erweiterbaren Ökosystems darstellt, kommt es zwangsläufig zu einer Aufwertung ökologischer und sozialer Faktoren und damit auch zu einer Neubewertung der unternehmerischen Standortwahl bei erneuerbaren Energien.

Ellis et al. (2007) und Aitken (2010a) konnten aufzeigen, dass gerade die Akzeptanz, die Bosch (2012:120) als den derzeit limitierenden Faktor beim Ausbau von erneuerbaren Energien bezeichnet, in großer Abhängigkeit zu ökologischen und sozialen Aspekten der Energiewende steht. Die
Erweiterung bestehender Ansätze um ökologische und soziale Standortparameter ist deshalb grundlegend auf dem Weg zu einer ausgewogenen Raumentwicklung.

\section{Problemstellung und Zielsetzung der Arbeit}

\subsection{Problemstellung}

Es ist festzustellen, dass die Haltung von Anwohnern gegenüber erneuerbaren Energien räumlich stark variiert. Das Spektrum reicht dabei von Energieregionen, in denen sich die Bürger engagiert an der Energiewende beteiligen (IdE, 2014:7), bis hin zu Gebieten, die eine Häufung von Bürgerinitiativen aufweisen, die sich gegen den weiteren Ausbau regenerativer Kraftwerke formieren und auf die fehlende räumliche Sensitivität von Standortentscheidungen verweisen (Becker et al., 2012a:52).

Die tatsächlichen Gründe für diese räumlichen Disparitäten sowie die Möglichkeiten der standortplanerischen Optimierung wurden nur unzureichend erörtert. Dies erscheint jedoch umso drängender, als das ehrgeizige Ziel der deutschen Bundesregierung, den Energieanteil an der Stromerzeugung aus erneuerbaren Energien auf $45 \%$ bis 2025 und auf $60 \%$ bis 2035 auszubauen (Die Bundesregierung, 2015), die ländlichen Räume Deutschlands künftig noch stärker beanspruchen wird, als dies bislang der Fall war.

\subsection{Zielsetzung}

Ziel der Studie ist es, einen Beitrag zu einem besseren Verständnis der Grundlagen für einen raumverträglichen Ausbau von erneuerbaren Energien auf regionaler Maßstabsebene zu leisten.

In einem ersten Schritt werden hierzu zentrale Standortparameter eines raumverträglichen Ausbaus regenerativer Technologien theoretisch aus dem bisherigen Stand der Forschung hergeleitet und begründet. In einem zweiten Schritt gilt es, dieses Instrumentarium konkret auf eine Planungsregion anzuwenden, um hierdurch eine vergleichende Analyse mit der tatsächlichen räumlichen Verteilung von erneuerbaren Energien zu ermöglichen. Sollte das tatsächliche Standortmuster schließlich von jenem abweichen, das aus theoretischer Perspektive einen raumverträglichen Ausbau erneuerbarer Energien garantieren würde, so kann festgestellt werden, welche Standortparameter unberücksichtigt geblieben sind und so einem suboptimalen Standortmuster möglicherweise Vorschub geleistet haben. In einem letzten Schritt wird überprüft, inwieweit der entwickelte theoretische Ansatz tatsächlich Hinweise auf regional bedeutsame Standortparameter liefern kann. Die Einbeziehung des Faktors Zeit ist dabei von großer Relevanz, da es keinen Endzustand einer optimalen räumlichen Verteilung von erneuerbaren Energien gibt, sondern das Optimum stets als Spiegel der gegenwärtig bestehenden wirtschaftlichen, gesellschaftlichen und technologischen Rahmenbedingungen zu verstehen ist. Aus diesem 
Grund werden auch Szenarien einer raumverträglichen Energiewende erstellt.

Die Umsetzung erfolgt beispielhaft anhand des Ausbaus von Wind- (WKA), Photovoltaik- (PV), Biogas- (BGA) und Erdwärmekraftwerken/Tiefengeothermie (EWK) in der bayerischen Planungsregion 9 (Augsburg). Da das Ausbaupotenzial der Wasserkraft innerhalb der Untersuchungsregion weitgehend ausgeschöpft ist, wird dieser Technologiepfad ausgeklammert. Generell ist zu erwähnen, dass die Bayerische Staatsregierung die Bemühungen um die Energiewende nach den Ereignissen von Fukushima im März 2011 erheblich forciert hatte, und zwar mittels des Energiekonzeptes „Energie innovativ“. Speziell der Windenergie wurden hierdurch zunächst größere räumliche Möglichkeiten als zuvor eingeräumt (StMI et al., 2011). Im Jahr 2014 wurde dies jedoch revidiert, indem der Regelabstand zwischen Wohnsiedlungen und Windrädern auf zwei Kilometer festgelegt wurde (Scharnitzky et al., 2014:33).

\section{Theoretisches Standortoptimum erneuerbarer Energien}

\subsection{Grenzen bisheriger Standortkonzepte}

Die Standortplanung für Wind-, PV-, Biogas- und Erdwärmekraftwerke zeichnet sich bislang dadurch aus, dass Fragen zur räumlichen Optimierung nur innerhalb des jeweiligen Technologiepfades, d. h. unabhängig von den Potenzialen und Flächenansprüchen der übrigen erneuerbaren Energien, erörtert werden. Stellvertretend hierfür stehen die Studien zur Wind- (Iwes, 2011; Ludwig und Bosch, 2014), Bio- (Starick et al., 2011; Schardinger et al., 2012) bzw. Solarenergie (Kallmünzer, 2006; Zink, 2010) sowie zur Erdwärme (Paschen et al., 2003; Wenzel et al., 2009). Der technologiespezifische Standortentscheidungsprozess ist dabei undifferenziert, wird der ländliche Raum doch lediglich auf die Kategorien Gunst- und Ungunstraum reduziert, wie dies beispielhaft anhand der oft zitierten, wenig aussagekräftigen Standortkarte zum Erdwärmepotenzial in Deutschland nachvollzogen werden kann, die sowohl das Norddeutsche Tiefland als auch weite Teile Süddeutschlands in die gleiche Raumkategorie günstiger petro- sowie hydrothermaler Voraussetzungen zwängt (Wenzel et al., 2009:24), ohne dabei mögliche Interferenzen zu diagnostizieren.

Diese Art der Regionalisierung, die sich primär auf natürliche Standortfaktoren bezieht, spiegelt nicht nur akteursbezogene Präferenzen wider (Gailing et al., 2013:21ff.), deren große Vielfalt den Standortfindungsprozess verzerrt, sondern ist darüber hinaus einseitig von ökonomischen Überlegungen geprägt (Prinz et al., 2009). Die ökologische und soziokulturelle Vielfalt ländlicher Räume wird dadurch ignoriert. Aus diesem Grund werden im weiteren Verlauf der Studie, neben ökonomischen, auch ökologische und soziale Standortparameter eingeführt (siehe Punkt 3.4).
Geographische Informationssysteme, wie der EnergieAtlas Bayern (StMWI, 2012), liefern zwar Informationen zu ökologischen und sozialen Standortparametern (bspw. Eignungs- und Tabuzonen). Als ernsthafte Entscheidungsgrundlage für einen konkreten Ausbau von erneuerbaren Energien auf kommunaler und regionaler Ebene können derartige Instrumente jedoch nicht fungieren, da die verfügbaren Informationen zu oberflächlich sind. Die steuernde Wirkung der Raumordnung ist bislang ebenfalls begrenzt, denn Einig et al. (2011) erkennen, dass der Windenergie seitens der Raumplanung einerseits zu wenig Flächen bereitgestellt werden, andererseits das Zustandekommen der regionalen Raummuster auf arbiträren Entscheidungsprozessen zu basieren scheint. Schöbel (2012:22) kritisiert des Weiteren die destruktive Raumplanungskultur, die fordere, dass erneuerbare Energien nur mehr auf Restflächen, mit z. T. erheblicher Vorbelastung, ausgebaut werden dürfen. Dies widerspreche dem Grundsatz des Raumordnungsgesetzes, gleichwertige Lebensverhältnisse in ländlichen Räumen herzustellen.

\subsection{Neues Verhältnis zwischen Standort und Technologie}

Entsprechend der Zielsetzung ist es notwendig, den Begriff „Raumverträglichkeit“ zu operationalisieren. Hierzu muss ein Bewertungsschema entworfen werden, innerhalb dessen einerseits für Teilräume jeweils spezifische ökonomische, ökologische und soziale Anforderungen an die regenerativen Technologien definiert werden, andererseits die erneuerbaren Energien entsprechend ihrer Eignung für eben diese Teilräume klassifiziert werden. Erst dadurch wird eine raumverträgliche Zuordnung von erneuerbaren Energien möglich.

Entscheidend dabei ist, sich von der vorherrschenden Denkart der isolierten Standortplanung zu lösen, die den optimalen Standort für eine von vornherein festgelegte erneuerbare Energieform zu identifizieren versucht. Vielmehr geht es darum, für einen gegebenen Standort bzw. Raum die passende erneuerbare Energie zu finden. Bathelt und Glückler (2002:93f.) schreiben: „Interessanter als die Frage nach dem Standort bei einer vorgegebenen Nutzung ist deshalb die Frage nach der konkreten Landnutzung zu einem vorgegebenen Standort [...].“ Im Sinne einer ,,energetischen Landnutzungslehre“ wird die Idee v. Thünens so auf den Ausbau von erneuerbaren Energien übertragen. Charakteristisch dabei ist, dass nicht mehr ein Standort-, sondern ein Technologieentscheidungsprozess durchgeführt wird, an dessen Ende jene Energieform identifiziert wird, die im Rahmen des Zusammenspiels mehrerer Standortparameter eine verträgliche Raumnutzung verspricht.

\subsection{Herleitung zentraler Raumtypen}

Der neue Ansatz zeichnet sich dadurch aus, dass für den Raum Anforderungen an die erneuerbaren Energien defi- 
niert werden. Der Anforderungskatalog wird jedoch entsprechend der regional unterschiedlichen Voraussetzungen variieren. Hieraus erwächst die Notwendigkeit, Teilräume zu bilden, die sich hinsichtlich ihrer Anforderungen an den Ausbau von erneuerbaren Energien voneinander unterscheiden. Bei der Bildung von Teilräumen wird die Empfindlichkeit eines Raumes gegenüber Eingriffen als Klassifizierungsmerkmal herangezogen. Grundlegend für die Regionalisierung ist die Klassifizierung von Bosch (2012:152ff.), die folgende Teilräume innerhalb der Untersuchungsgebiete definiert:

a. Vorbelasteter Raum: In diesen Raum wurde verkehrsinfrastrukturell bereits stark eingegriffen, so dass jegliche weitere Überformung durch erneuerbare Energien zu keiner nennenswerten Abwertung mehr führen kann. Zum Vorbelasteten Raum zählen auch Konversionsflächen. Bedeutend ist, dass es sich um eine Fläche handelt, die aus ökologischer Perspektive noch stark unter den Folgen der ehemals militärischen oder wirtschaftlichen Nutzung zu leiden hat. Die Sensibilität des Vorbelasteten Raumes gegenüber einer Technisierung ist sehr gering. Als Grundlage für den Vorbelasteten Raum wurden für den Bereich Verkehrsinfrastruktur OpenStreetMap-Daten verwendet, wobei Autobahnen, Bundesstraßen und Schienenwege mit jeweils einem Kilometer gepuffert wurden, da die Vorbelastung über den reinen Flächenumfang der einzelnen Raumelemente hinausreicht. Die Konversionsflächen wurden mit Hilfe von GoogleEarth erhoben und digitalisiert.

b. Forstwirtschaftlicher Raum: Dieser Raumtyp beinhaltet die Flächenkategorien Laub-, Nadel- und/oder Mischwälder. Es geht darum, Räume zu identifizieren, die aufgrund eines höheren Anteils an Waldgebieten weniger Flächen für die Errichtung von erneuerbaren Energien zur Verfügung stellen als andere Räume bzw. die Möglichkeit bieten, Technologien im Sichtschutz eines Waldes zu platzieren. Beispielhaft hierfür stehen die Arbeiten von Tobias et al. (2003) sowie Ludwig und Bosch (2014). Handelt es sich um ein Waldgebiet einer hohen Schutzkategorie (bspw. ein Waldschutzgebiet), so wird dieser Raum nicht dem Forstwirtschaftlichen, sondern dem Verletzlichen Raum (s. u.) - d. h. der empfindlicheren Raumkategorie - zugeordnet. Die Sensibilität des Forstwirtschaftlichen Raumes gegenüber einer Technisierung ist gering. Als Datengrundlage für den Fortwirtschaftlichen Raum fungiert der CorineLandcover-Datensatz (Keil et al., 2010).

c. Landwirtschaftlicher Raum: Dieser Raumtyp beinhaltet Acker- und Grünland. Daher kommt der Nahrungs- und Futtermittelproduktion dort eine große Bedeutung zu. Es ist davon auszugehen, dass dieser Raumtyp durch die überwiegend hohe Intensität der landwirtschaftlichen Bewirtschaftung (vgl. landwirtschaftliches Intensivgebiet Donau-Ries) ökologisch gefährdet ist (Boden- erosion, Eutrophierung etc.) (Bosch, 2010). Die Sensibilität des Landwirtschaftlichen Raumes gegenüber einer Technisierung ist als „,mittel“ einzustufen. Als Datengrundlage für den Landwirtschaftlichen Raum fungiert der Corine-Landcover-Datensatz.

d. Dienstleistungsraum: Dieser Raumtyp zeichnet sich durch ein schützenswertes Landschaftsbild aus. Bedeutende kulturlandschaftliche Elemente prägen den Raum, die in engem Zusammenhang mit der Erholungsfunktion stehen. Die Sensibilität des Dienstleistungsraumes gegenüber einer Technisierung ist hoch. Der Dienstleistungsraum wird aus folgenden Elementen der Kulturlandschaft aufgebaut: Kapellen, Kirchen, Wallfahrtsorte, Klöster, Synagogen, Burgställe, Burgruinen, Burgen, Schlösser, Naturdenkmäler und Geotope.

Entscheidend für die kartographische Visualisierung des Dienstleistungsraumes ist, dass dieser sich nicht nur aus den relativ geringen Flächenausmaßen der Bauwerke selbst, sondern zusätzlich aus den Puffern um diese Bauwerke, mit unterschiedlichen Radien - entsprechend ihrer kulturlandschaftlichen Bedeutung zwischen 500 und $2500 \mathrm{~m}-$, zusammensetzt. Dies ist dadurch zu erklären, dass die landschaftliche Prägung der Kulturlandschaftselemente nicht lokal beschränkt ist, sondern eine größere Reichweite hat. Als Datenquelle fungierten das Bayerische Landesamt für Denkmalpflege, die Landratsämter des Untersuchungsraumes sowie das Landesamt für Umwelt.

e. Verletzlicher Raum: Dieser Raumtyp umfasst besonders schützenswerte Gebiete. Er ist nicht nur bedeutend als Erholungsraum. Vielmehr stellt der Verletzliche Raum einen Lebensraum für gefährdete Flora und Fauna dar. Die Sensibilität des Verletzlichen Raumes gegenüber einer Technisierung ist daher als sehr hoch einzustufen. Der Verletzliche Raum wird aus den Fauna-Flora-Habitat-Gebieten (Natura 2000), SPAVogelschutz-Gebieten (Natura 2000), Naturschutzgebieten, Landschaftsschutzgebieten, Nationalparken sowie Ramsar-Gebieten (Feuchtgebiete) aufgebaut. Als Datengrundlage dienen die frei zugänglichen Vektordatensätze des Bayerischen Landesamts für Umwelt.

Abbildung 1 zeigt die räumliche Verteilung der einzelnen Raumtypen in der Planungsregion 9. Charakteristisch sind v. a. der Vorbelastete Raum, der sich linienhaft über die Region erstreckt, sowie der Dienstleistungsraum, der inselartig den Raum durchdringt. Für den speziellen Fall, dass zwei Raumtypen überlappen, werden die Flächen jenem Raumtyp zugeordnet, der eine größere Empfindlichkeit gegenüber einer Technisierung durch erneuerbare Energien aufweist. Dadurch wird ein hoher Grad an Raumverträglichkeit erreicht. 


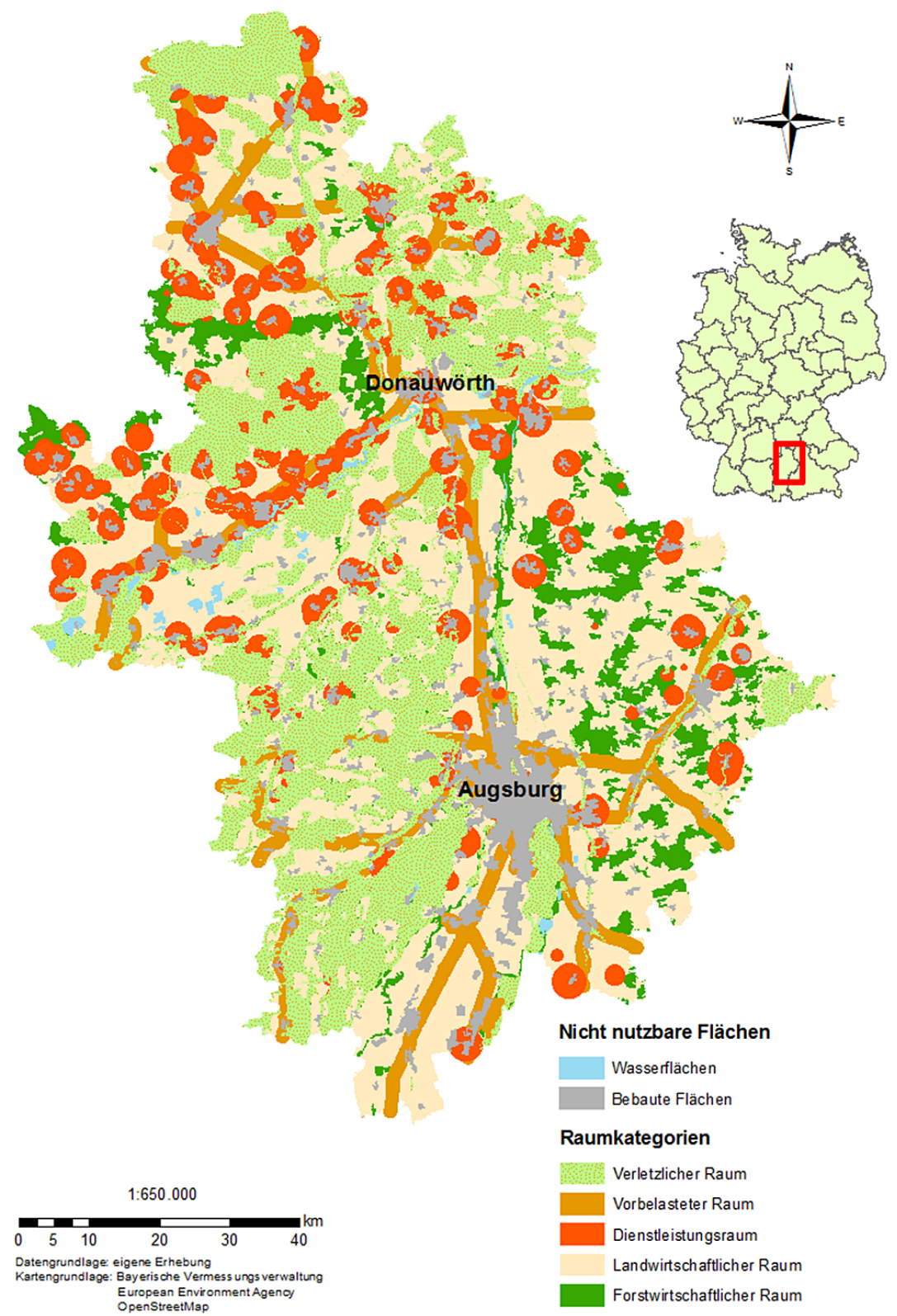

Abb. 1. Raumtypisierung der Planungsregion 9.

\subsection{Herleitung zentraler Standortparameter}

Auf der Suche nach einem geeigneten Parameterkatalog zur Bewertung der Standortqualität des Ausbaus von erneuerbaren Energien ist jene Literatur miteinzubeziehen, die den Fokus auf urbane Räume richtet. Dies mag zunächst verwundern, handelt es sich bei der flächenintensiven Energiewende doch vorwiegend um ein Phänomen jenseits der dicht besiedelten Ballungsräume (Birkner, 2013:227), die nach Gailing und Röhring (2015:32) eher als Standortsuchraum für konventionelle Kraftwerkseinheiten fungieren und aufgrund der stark eingeschränkten Flächenverfügbarkeit in- nerhalb der Stadtgrenzen (Gailing et al., 2013:24) fast nur gebäudeintegrierte Lösungen erlauben (Porsche, 2010:666).

Doch die Eignung urbaner Energiekonzepte basiert gerade auf der ungleich hohen Interaktionsdichte, der stark ausgeprägten Flächennutzungskonkurrenzen und der kleinsträumig variierenden Standortvoraussetzungen. Ein ausgeklügeltes urbanes Energiekonzept muss daher ein hohes $\mathrm{Ma}$ an Raumverträglichkeit aufweisen, um langfristig bestehen zu können (BMVBS et al., 2008 und 2009; Ecofys, 2008). Standortkonzepte ländlicher Räume sollten sich daher an jenen urbaner Räume orientieren. Nicht zuletzt sind die für den urbanen Raum gewählten Standortparame- 
ter Gestehungskosten, Flächeneffizienz, Regelbarkeit, Umweltfreundlichkeit, Rückbaufähigkeit und Genehmigungsrecht auch auf den ländlichen Raum übertragbar (BMVBS et al., 2009:31ff.). Darüber hinaus müssen weitere Parameter miteinbezogen werden, die das Landschaftsbild, Fragen der Energiegovernance sowie die Betriebsdauer von regenerativen Technologien betreffen (Bosch, 2012:160ff.).

Im Folgenden werden die zentralen Standortparameter einerseits hergeleitet und begründet, andererseits nach ihrer ökonomischen, ökologischen oder sozialen Natur geordnet. Diese Klassifikation hilft im Rahmen der später durchzuführenden vergleichenden Analyse leichter zu begreifen, welche Standortparameter nicht berücksichtigt wurden und so möglicherweise zu einer suboptimalen Raumstruktur beigetragen haben.

\subsection{1 Ökonomische Parameter}

- Gestehungskosten: Dabei werden jeweils die Gesamtkosten auf die produzierte Nettoenergie bezogen (ISE, 2013). Kaltschmitt et al. (2006:16) bezeichnen die Kosten als ,wesentliche Kenngröße jeder Möglichkeit zur Energiebereitstellung“. Eine genaue Darstellung der zu erwartenden Kosten ist nicht zuletzt deswegen notwendig, da die z. T. unerfahrenen, neu am Energiemarkt auftretenden Akteure, wie Privatpersonen (Klagge und Brocke, 2013:12), nicht immer mit den wirtschaftlichen Risiken von Energie-Projekten vertraut sind (Böttcher, 2009; Herbes und Friege, 2015) und oftmals riskante Standortoptionen ins Planungskalkül miteinbeziehen. Die Einführung eines aussagekräftigen monetären Standortparameters ist daher angebracht.

- Regelbarkeit: Anhand dieses Parameters wird untersucht, inwieweit sich eine Technologie zur Deckung von Grund- und Spitzenlast eignet, denn Anlagenbetreiber können den produzierten Strom auch unabhängig von der EEG-Vergütung direktvermarkten und dem Regelenergiemarkt zur Verfügung stellen (Kopp et al., 2012:244ff.). Hierdurch ist es möglich, sich dem Risiko degressiver Vergütungssätze zu entziehen und unabhängig von staatlichen Fördermechanismen für ökonomische Stabilität zu sorgen. Die Relevanz eines Regelenergiemarktes rührt daher, dass die Einspeisungen von Wind- und Solaranlagen aufgrund ihres intermittierenden Charakters eine hohe zeitliche und räumliche Variabilität aufweisen (Bosch, 2013:413).

- Volllaststunden: Damit in Zusammenhang steht die jährliche Betriebsdauer, die über die Bandbreite der zur Verfügung stehenden Technologien stark variiert. Von den 8766 h des Jahres 2012, waren in Deutschland Photovoltaik- nur $980 \mathrm{~h}$, Windenergie- $1530 \mathrm{~h}$ und Biomasseanlagen immerhin $6040 \mathrm{~h}$ mit Volllast am Netz (BDEW, 2013:21). Diese Unterschiede, die regional nochmals Ausprägungen annehmen können, die erheblich vom bundesdeutschen Durchschnitt abweichen, sind von großer ökonomischer Relevanz und daher als Standortparameter zu berücksichtigen.

\subsection{2 Ökologische Parameter}

- Flächeneffizienz: Brücher (2008:4) betont, dass die Energiedichten regenerativer Energieträger wesentlich geringer sind, als diejenigen fossil-nuklearer Energiesysteme. Eine mittlere globale Strahlungsenergie auf der Erdoberfläche von $0,59 \mathrm{~kW} \mathrm{~m}^{-2}$ ist nicht vergleichbar mit den großen Energiedichten ergiebiger Ölfelder (Bosch, 2013:398). Doch auch innerhalb des Spektrums regenerativer Quellen gibt es Unterschiede in den Energiedichten. In intensiv genutzten Räumen kann es daher entscheidend sein, jene erneuerbaren Energien einzusetzen, deren Flächenansprüche mit den Ausmaßen regionaler Ökosystemverbunde übereinstimmen. Doch auch für äußerst sensible Teilräume, innerhalb derer die Flächenbelastung auf ein Minimum reduziert werden soll, ist die Kenntnis der technologiespezifischen Flächeneffizienz - vgl. Peyke et al. (2013:23) - von großer Relevanz.

- Rückbaufähigkeit: Bei den erneuerbaren Energien handelt es sich im Allgemeinen um Technologien, die sich relativ leicht und schnell rückbauen lassen. Doch verweisen BMVBS et al. (2009:42) darauf, dass dennoch zwischen aufwändigen, permanenten sowie temporären, leicht rückbaufähigen Technologien zu unterscheiden ist, mit entsprechenden raumzeitlichen Folgen für genutzte und angrenzende Ökosysteme. Gerade für sensible Teilräume sollten Technologien eingesetzt werden, die sich leicht rückbauen lassen.

- Ökobilanz: Energierücklaufzeit, Energiebilanz, Emissionen von Treibhausgasen, Luftschadstoffen und Giftstoffen, Möglichkeiten des Rezyklierens sowie das Störfallpotenzial bestimmen die Umweltfreundlichkeit einer erneuerbaren Energie und können zu einem wichtigen ökologischen Standortparameter werden. Zepf et al. (2014) haben in ihrer Studie gezeigt, dass dem materiellen Aufbau regenerativer Technologien eine große Vielfalt an Ressourcen zu Grunde liegt, die z. T. ökologisch bedenklich sind und daher in eine ganzheitliche Standortbewertung miteinzubeziehen sind.

\subsubsection{Soziale Parameter}

- Landschaftsintegration: Bei diesem Parameter gilt es zu untersuchen, inwieweit sich eine regenerative Technologie ins Landschaftsbild einfügen lässt. Dabei spielen Aspekte wie Ästhetik, Auffälligkeit, Überdeckung, Eigenartverluste und Erholungseignung eine Rolle. Mittlerweile existieren zahlreiche Studien, die sich kritisch 
Tabelle 1. Spezifische Raumanforderungen: gering (-), mittel (0), hoch (+).

\begin{tabular}{|l|c|c|c|c|c|}
\hline & Verletzlicher Raum & Dienstleistungsraum & Landwirtschaftlicher Raum & Forstwirtschaftlicher Raum & Vorbelasteter Raum \\
\hline Flächeneffizienz & 0 & 0 & 0 & + & - \\
\hline Genehmigung & + & + & 0 & 0 & - \\
\hline Gestehungskosten & - & 0 & 0 & - & + \\
\hline Landschaftsintegration & + & + & 0 & - & - \\
\hline Ökobilanz & + & + & - & - & - \\
\hline Partizipation & + & 0 & 0 & 0 & + \\
\hline Regelbarkeit & - & - & 0 & 0 & + \\
\hline Rückbaufähigkeit & + & + & 0 & + & + \\
\hline Volllaststunden & - & 0 & & \\
\hline
\end{tabular}

mit dem wenig landschaftsgerechten Ausbau von erneuerbaren Energien auseinandersetzen, wie die Arbeiten von Jedicke (1997), Peters et al. (2009), Nohl (2010), Scheidler (2010), Schöbel (2012), Megerle (2013), Zimmerer (2013) sowie Kirchhoff (2014) belegen. Es ist zwar davon auszugehen, dass künftige Generationen erneuerbare Energien als vertraute Elemente heimatlicher Normallandschaft wahrnehmen werden (Kühne, 2011). Jedoch entbindet der Gewöhnungseffekt nicht von der Verantwortung einer landschaftlich angepassten Integration.

- Partizipation: In der wissenschaftlichen Literatur, in der es um Fragen der Energiegovernance geht - vgl. Kanning et al. (2009), Aitken (2010b), Kammer (2011), Becker et al. (2012a und 2012b), Jakubowski (2012), Heinrichs (2013), Klagge und Arbach (2013), Gailing et al. (2013), Moss et al. (2013) sowie Gailing und Röhring (2015) -, wird verdeutlicht, dass die innere Struktur des deutschen regenerativen Energiesystems - also Einspeisevorrang, Vergütungsgarantie, kleine Kraftwerkseinheiten mit großem Flächenverbrauch, Standorte in Verbrauchernähe - zu einer Logik der Dezentralität geführt hat, mit entsprechenden Möglichkeiten zur Partizipation für eine große Anzahl an Akteuren. Die erneuerbaren Energien unterscheiden sich jedoch dahingehend, inwieweit sie Möglichkeiten der bürgerlichen Mitgestaltung erlauben. Dies gilt es im Rahmen eines Standortparameters zu berücksichtigen.

- Genehmigungsrecht: Heindl et al. (2014:508) betonen, dass bestimmte soziale Gruppen als Verlierer der Energiewende gelten können. Kühne (2011:296) beschreibt, dass nachhaltige Entwicklung per se eine starke Verbindung zum Sozialen enthält. Aitken (2010a) betont, dass dieser Zusammenhang unterschätzt oder nicht verstanden wird, wodurch sich die Akzeptanz der Energiewende verringert (Ellis et al., 2007). Der starke Ausbau von erneuerbaren Energien ist demnach kritisch zu hinterfragen, wenn Genehmigungsverfahren nicht die legitimen Anliegen aller sozialen Gruppen respektieren.
Damit werden Genehmigungsverfahren - als ein ursprünglich immanent ökologischer sowie einem daraus ableitbaren ökonomischen Standortparameter, wenn bspw. die Dauer eines Verfahrens und damit seine Kosten betrachtet werden - auch zu einem Standortparameter mit sozialer Komponente. Genehmigungsverfahren zu erneuerbaren Energien werden durch BauGB, BImSchG, BNatSchG, Wasser- und Bergrecht sowie Umweltverträglichkeitsprüfungen festgelegt.

Nachdem die zentralen Standortparameter hergeleitet wurden, stellt sich die Frage nach der jeweiligen Relevanz für die unterschiedlichen Raumtypen. Hierzu wurde eine Gewichtung gewählt, die den Gegebenheiten sensibler Teilräume dadurch Rechnung trägt, indem ökologische und soziale Parameter eine größere Bedeutung haben, als ökonomische Parameter. Robusteren Räumen werden hingegen ökonomisch bessere Lösungen „zugemutet“, wodurch ökologisch-soziale Standortparameter zwangsläufig als nachrangig eingestuft werden (vgl. Tabelle 1).

\subsection{Raumverträgliches Standortmuster erneuerbarer Energien}

Nachdem die Anforderungskataloge für die Teilräume definiert wurden, gilt es herauszufinden, inwieweit die erneuerbaren Energien diesen Anforderungen Rechnung tragen können. Hierzu ist es erforderlich, die ökonomischen, ökologischen und sozialen Eigenschaften der erneuerbaren Energien zu erfassen und zu vergleichen. Es ist zu konstatieren, dass sich die erneuerbaren Energien diesbezüglich erheblich voneinander unterscheiden und jede Technologie spezifische Vor- und Nachteile aufweist.

Im Rahmen der Studie werden diese Unterschiede aus Gründen der Vereinfachung auf drei Klassen (,,-“, „, ,“, ,+“") reduziert. Tabelle 2 zeigt die Klassifizierung der erneuerbaren Energien unter den aktuellen technologischen Rahmenbedingungen. Anhand des Parameters Flächeneffizienz wird die Klassifizierung beispielhaft erläutert:

Laut BMVBS et al. (2009:35) benötigen Biogasanlagen zwischen 45 und 250 ha, um eine Gigawattstunde Strom pro 
Tabelle 2. Spezifische Technologievoraussetzungen: schlecht (-), mittel (0), gut (+).

\begin{tabular}{|l|c|c|c|c|}
\hline & Biomasse & Geothermie & Photovoltaik & Windkraft \\
\hline Flächeneffizienz & - & + & 0 & 0 \\
\hline Genehmigung & 0 & - & + & + \\
\hline Gestehungskosten & 0 & - & 0 & + \\
\hline Landschaftsintegration & + & + & - & 0 \\
\hline Ökobilanz & 0 & 0 & + & + \\
\hline Partizipation & 0 & - & - & + \\
\hline Regelbarkeit & + & + & + & - \\
\hline Rückbaufähigkeit & - & + & - & 0 \\
\hline Volllaststunden & + & + & & 0 \\
\hline
\end{tabular}

Tabelle 3. Zuordnung von Räumen und Technologien.

\begin{tabular}{|c|c|c|c|c|c|}
\hline & Verletzlicher Raum & Dienstleistungsraum & Landwirtschaftlicher Raum & Forstwirtschaftlicher Raum & Vorbelasteter Raum \\
\hline Flächeneffizienz & EWK, PV, WKA & EWK, PV, WKA & EWK, PV, WKA & EWK & BGA, EWK, PV, WKA \\
\hline Genehmigung & PV, WKA & PV, WKA & BGA, PV, WKA & BGA, PV, WKA & BGA, EWK, PV, WKA \\
\hline Gestehungskosten & BGA, EWK, PV, WKA & BGA, PV, WKA & BGA, PV, WKA & WKA & WKA \\
\hline Landschaftsintegration & BGA，EWK & BGA, EWK & BGA, EWK, PV, WKA & BGA, EWK, PV, WKA & BGA, EWK, PV, WKA \\
\hline Ökobilanz & WKA & WKA & BGA，EWK，WKA & BGA, EWK, WKA & BGA, EWK, PV, WKA \\
\hline Partizipation & PV, WKA & BGA, PV, WKA & BGA, EWK, PV, WKA & BGA，EWK，PV, WKA & BGA, PV, WKA \\
\hline Regelbarkeit & BGA, EWK, PV, WKA & BGA, EWK, PV, WKA & BGA, EWK & BGA，EWK & BGA, EWK \\
\hline Rückbaufähigkeit & PV & PV & PV, WKA & PV, WKA & BGA, EWK, PV, WKA \\
\hline Volllaststunden & BGA, EWK, PV, WKA & BGA, EWK, WKA & BGA, EWK, WKA & BGA，EWK & BGA, EWK \\
\hline Summe: & $\begin{array}{c}7 \times P V \\
7 \times W K A \\
5 \times E W K \\
4 \times B G A\end{array}$ & $\begin{array}{c}7 \times \text { WKA } \\
6 \times \mathrm{PV} \\
5 \times \mathrm{BGA} \\
4 \times \mathrm{EWK}\end{array}$ & $\begin{array}{c}8 \times \text { WKA } \\
7 \times \text { BGA } \\
6 \times \text { EWK } \\
6 \times \text { PV }\end{array}$ & $\begin{array}{c}6 \times \text { BGA } \\
6 \times \mathrm{EWK} \\
6 \times \mathrm{WKA} \\
4 \times \mathrm{PV}\end{array}$ & $\begin{array}{c}8 \times \mathrm{BGA} \\
7 \times \mathrm{EWK} \\
7 \times \mathrm{WKA} \\
6 \times \mathrm{PV}\end{array}$ \\
\hline
\end{tabular}

Jahr zu erzeugen. Zur Produktion von einer Gigawattstunde Wärme werden zwischen 23 und 125 ha benötigt. Peters (2008:10) geht von 102 ha aus. Die Flächeneffizienz der Biogastechnologie wird daher als ,schlecht“ (-) eingestuft. Da die Energieübertragung bei Erdwärme unterirdisch erfolgt, ist die Flächeneffizienz „gut“ (+). Nach Angaben des BMVBS et al. (2009:33) beanspruchen Windkraftanlagen zwischen 3,7 und 11,1 ha, um jährlich eine Gigawattstunde Strom zu erzeugen. Peters (2008:10) geht von 5,7 ha aus, wobei nicht die reine Fundamentfläche, sondern die Abstandsfläche zur benachbarten Windanlage als Maß herangezogen wird. Die Flächeneffizienz der Windenergie - ,mittel“ (0) - liegt damit über jener von Biogas. Auch die Photovoltaik wird als ,mittel“ (0) eingestuft, denn nach Angaben des BMVBS et al. (2009:32) beanspruchen PV-Anlagen zwischen 3,0 und 9,3 ha, um jährlich eine Gigawattstunde Strom zu erzeugen. Peters (2008:10) geht von 4,4 ha aus.

Die erneuerbaren Energien werden nun dahingehend untersucht, inwiefern ihre Technologievoraussetzungen (vgl. Tabelle 2) den Raumanforderungen (vgl. Tabelle 1) entsprechen. Dabei wird so vorgegangen, dass jede Technologie, die den spezifischen Anforderungen, die für einen Teilraum definiert wurden, entsprechen kann, in die zugehörige Spalte eingetragen wird (vgl. Tabelle 3 ).
Beispielsweise können bzgl. des Parameters Flächeneffizienz drei Technologien, und zwar Erdwärmekraftwerke, PVund Windkraftanlagen, der Anforderung des Verletzlichen Raumes entsprechen. Jene Technologie, die in der Summe einer Spalte am häufigsten den Raumanforderungen entsprechen kann, wird schließlich dem jeweiligen Raum zugeordnet (vgl. Abb. 2).

In einem weiteren Schritt werden aus Abb. 2 nochmals jene Standorte extrahiert, auf denen die natürlichen Standortfaktoren tatsächlich einen wirtschaftlichen Betrieb der raumverträglichsten Technologie erlauben (vgl. Abb. 3). Die Studie geht von der Wirtschaftlichkeit der Windenergie bzw. Photovoltaik ab einer Windhöffigkeit von $5,5 \mathrm{~m} \mathrm{~s}^{-1}$ bzw. einer Globalstrahlung von mehr als $1150 \mathrm{kWh} \mathrm{m}^{-2}$ sowie einer Sonnenscheindauer von mehr als $1650 \mathrm{ha}^{-1}$ aus. Da die natürlichen Standortfaktoren bzgl. Biogas und Erdwärme über den gesamten Untersuchungsraum hinweg einen wirtschaftlichen Betrieb erlauben, wurden die Genehmigungsauflagen des Bundesimmissionsschutzgesetzes als raumdifferenzierende Größe herangezogen (BMJV, 2014).

\subsection{Räumliche Dynamik}

Von großer Bedeutung ist, dass sich strukturelle Umbrüche in Wirtschaft, Gesellschaft und Technologie regional sowie zeitlich differenziert in der Eignung von Flächen für den 


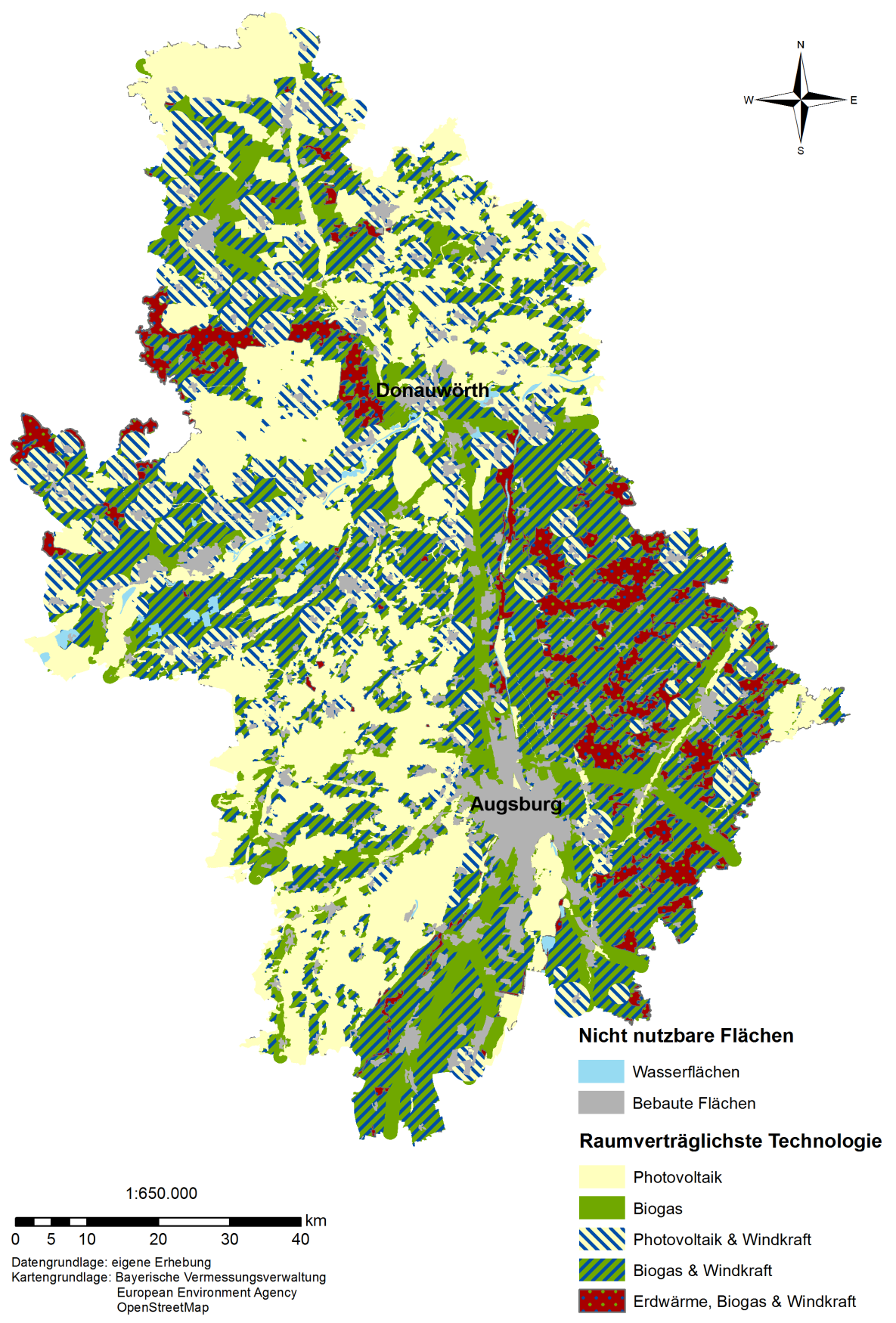

Abb. 2. Raumverträgliche Zuordnung von erneuerbaren Energien - aktueller technischer Stand.

Ausbau niederschlagen werden. Daher gilt es, die Standortpotenziale erneuerbarer Energien und insbesondere deren optimale räumliche Verteilung an potenzielle wirtschaftliche, gesellschaftliche und technologische Entwicklungspfade zu koppeln. Das Standortmodell muss die Möglichkeit bieten, Variationen dieser Rahmenbedingungen auf der Ebene alternativer Standortmuster abbilden zu können.

Es ist davon auszugehen, dass die unzähligen Kombinationsmöglichkeiten ökonomischer, ökologischer und sozia- ler Parameter auch unzählige Standortmuster einer optimalen Produktions- und Versorgungsstruktur mit sich bringen werden. Eine vorausschauende räumliche Planung greift diese Komplexität auf und versucht mittels Szenarien Korridore möglicher Entwicklungspfade abzustecken sowie die Robustheit potenzieller Standortmuster zu ermitteln. Auch Wettbewerbsmechanismen zwischen erneuerbaren Energien können so besser verstanden werden. 


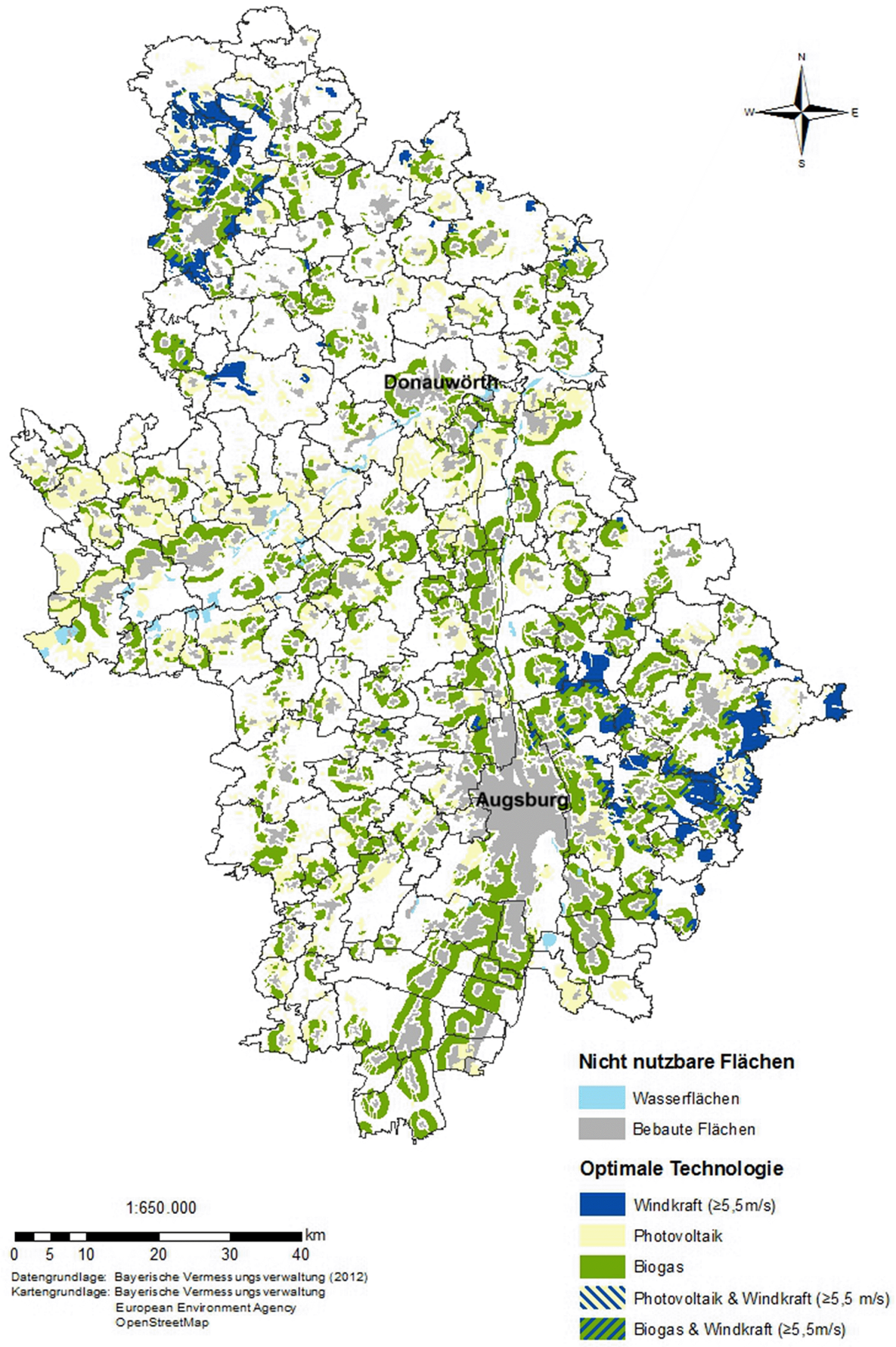

Abb. 3. Raumverträgliche Zuordnung von erneuerbaren Energien unter Einbeziehung optimaler natürlicher Standortfaktoren (z. B. 5,5 m s ${ }^{-1}$ Windenergie).

Beispielhaft wird das Szenario ,technologischer Fortschritt“ berechnet, um die große räumliche Dynamik der Energiewende zu illustrieren. Ausgehend von den aktuellen technologischen Rahmenbedingungen (vgl. Tabelle 2) wird im Sinne einer isolierenden Abstraktion angenommen, dass sich die Windkraft im Bereich der Parameter Genehmigung, Landschaftsintegration und Partizipation um jeweils eine Ka- tegorie verbessert. Es ist durchaus realistisch, dass Genehmigungsverfahren angepasst werden (StMI et al., 2011). Darüber hinaus arbeiten Landschaftsarchitekten an Konzepten zur besseren landschaftlichen Integration von Windkraftanlagen (Schöbel, 2012). Des Weiteren gibt es immer mehr Partizipationsmodelle, um Bürger finanziell und planerisch an der Windenergie zu beteiligen (Aitken, 2010b). 


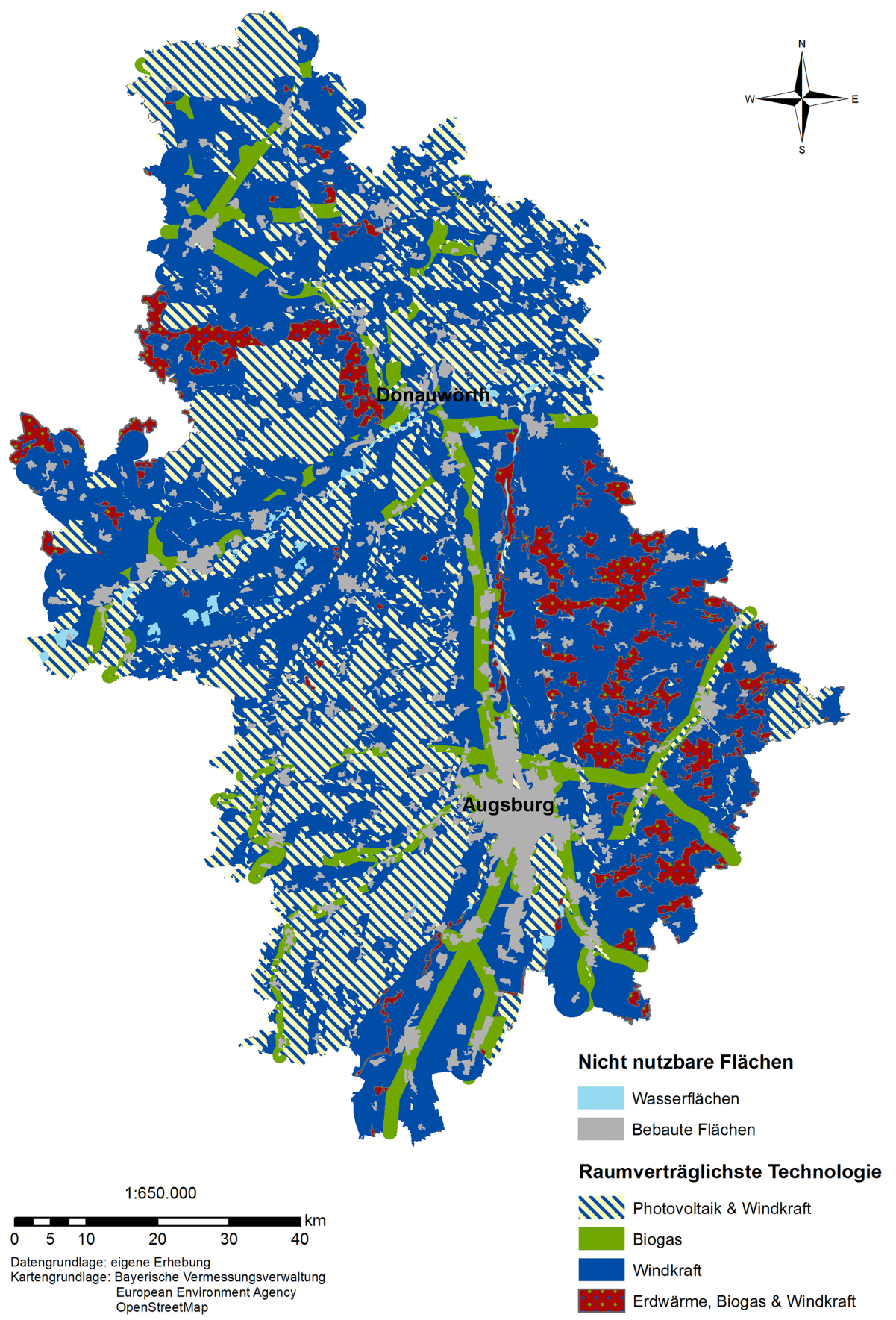

Abb. 4. Raumverträgliche Zuordnung von erneuerbaren Energien - Szenario „,technologischer Fortschritt“.

Die Variation der drei Parameter führt zu einer großen räumlichen Dynamik, denn die Windenergie steigt in weiten Teilen der Planungsregion 9 zur alleinigen raumverträglichsten Energieform auf (vgl. Landwirtschaftlicher Raum und Dienstleistungsraum) und verdrängt dabei Biogas und Photovoltaik (vgl. Abb. 4 mit Abb. 2). Im Forstwirtschaftlichen und Vorbelasteten Raum hingegen reagieren die angestammten Technologien robust auf die Änderungen der Rahmenbe- dingungen. Durch das neue Standortmuster ergibt sich unter Einbezug der natürlichen Standortfaktoren insgesamt ein schmalerer räumlicher Korridor für den Ausbau erneuerbarer Energien, da die nahezu umfassend raumverträgliche Windenergie auf relativ wenig wirtschaftliche Standortoptionen zurückgreifen kann (vgl. Abb. 5). Dies würde sich jedoch ändern, wenn sich durch technischen Fortschritt die Wirtschaftlichkeit der Windenergie nicht erst ab einer Windhöffigkeit 


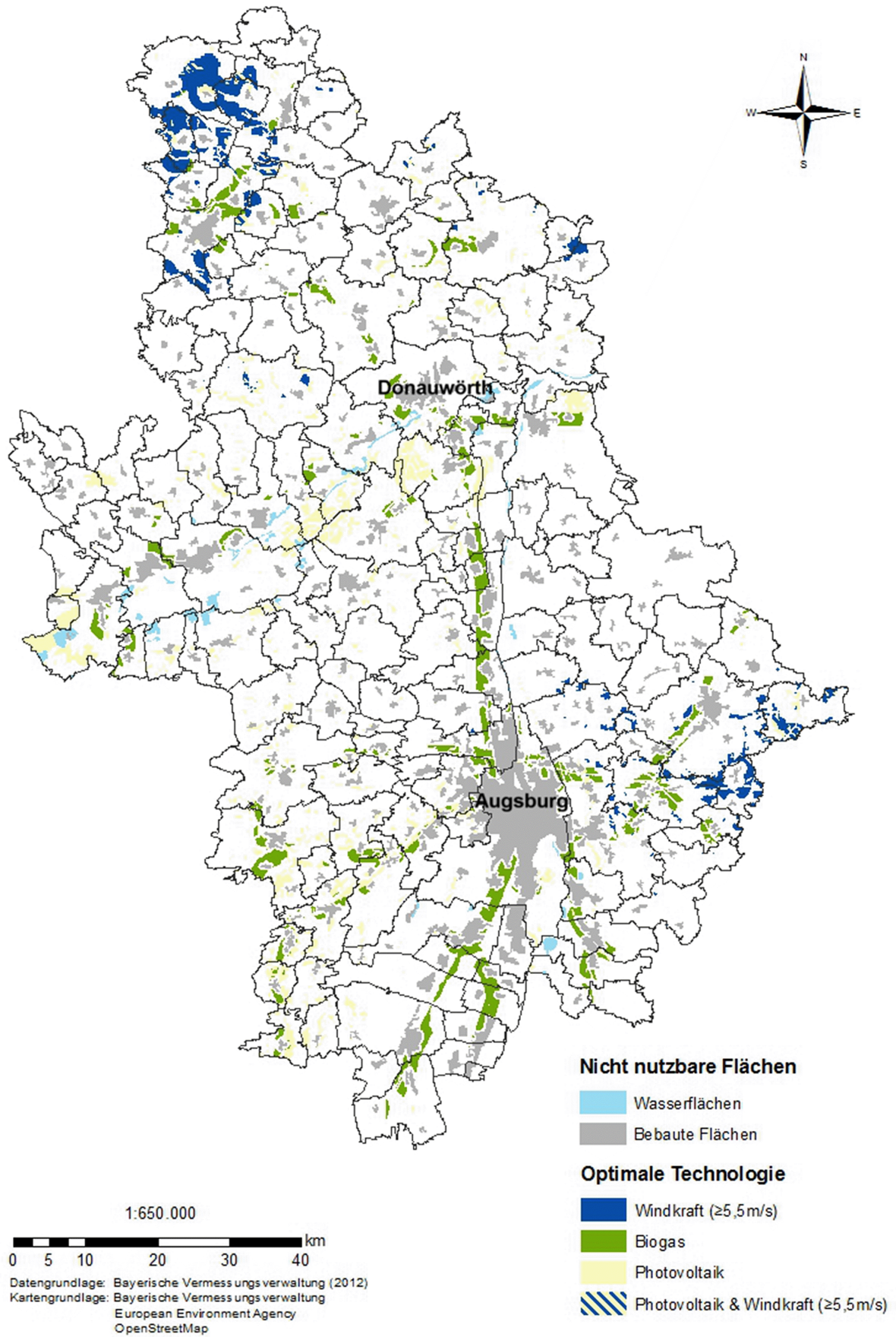

Abb. 5. Raumverträgliche Zuordnung von erneuerbaren Energien unter Einbeziehung optimaler natürlicher Standortfaktoren (z. B. 5,5 m s ${ }^{-1}$ Windenergie) - Szenario ,technologischer Fortschritt“.

von $5,5 \mathrm{~m} \mathrm{~s}^{-1}$, sondern bereits ab $5,0 \mathrm{~m} \mathrm{~s}^{-1}$ einstellen würde (vgl. Abb. 6).
4 Vergleichende Analyse - theoretisches Standortoptimum und tatsächliche Entwicklung

Nachdem das gegenwärtig raumverträgliche Standortmuster von erneuerbaren Energien ermittelt wurde (vgl. Abb. 2), erfolgt schließlich der Vergleich mit dem tatsächlichen Ausbau von Wind-, PV-, Biogas- und Erdwärmekraftwerken in der 


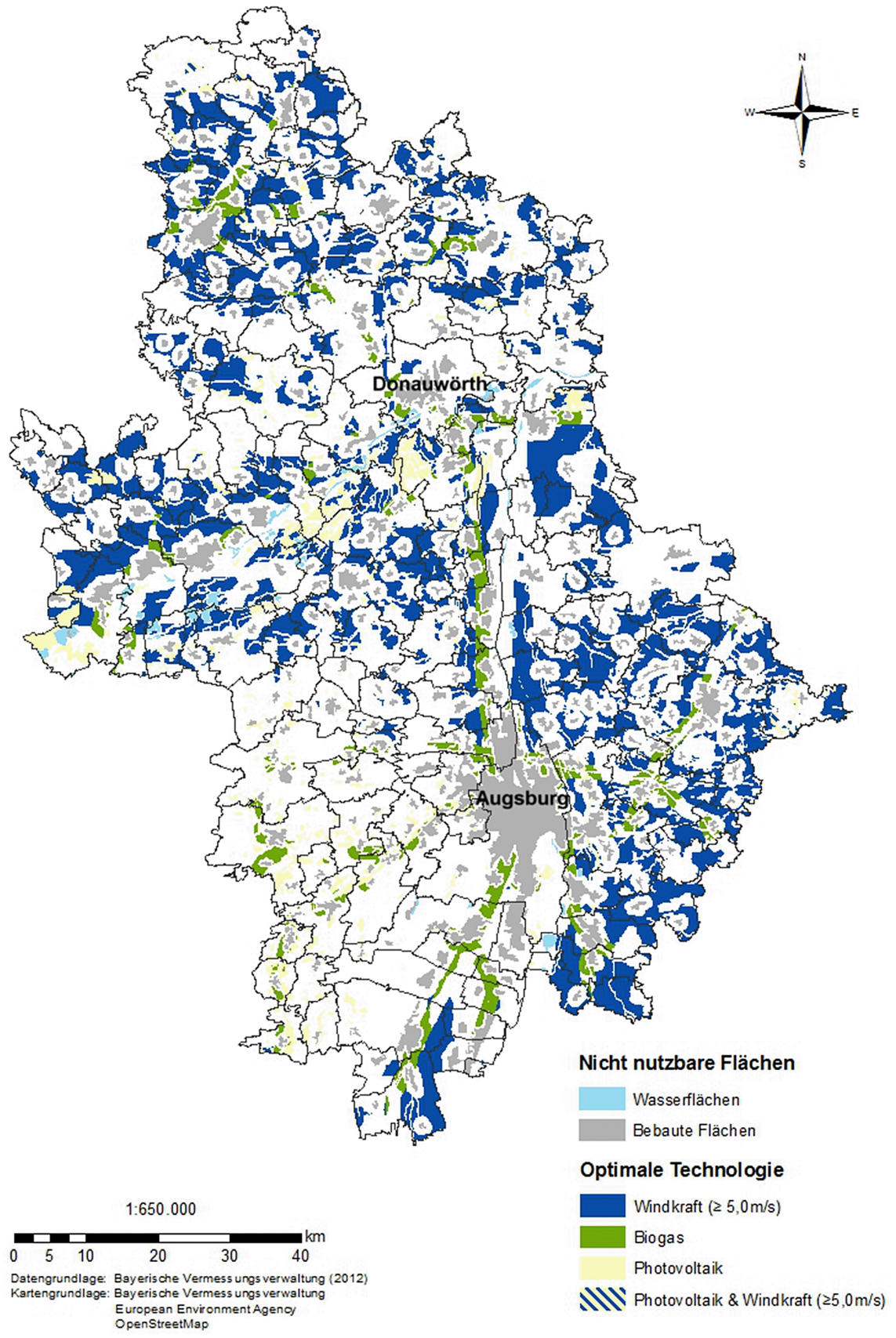

Abb. 6. Raumverträgliche Zuordnung von erneuerbaren Energien unter Einbeziehung optimaler natürlicher Standortfaktoren (z. B. 5,0 m s ${ }^{-1}$ Windenergie) - Szenario „technologischer Fortschritt“.

Planungsregion 9. Daraus lässt sich ableiten, inwieweit die bisherige Standortakquise raumverträglich war. Befindet sich eine erneuerbare Energie tatsächlich auf einem Standort, auf dem diese diejenige Technologie darstellt, die am häufigsten dem raumspezifischen Anforderungskatalog entsprechen konnte, so ist die Standortwahl raumverträglich. Stellt sie jedoch nur die zweit-, dritt- oder viertbeste Option dar, so ist die Standortentscheidung bedingt raumverträglich.
Abbildung 7 offenbart, dass v. a. die Standortakquise für PV-Freiflächenanlagen wenig raumverträglich ist. Kurios ist, dass gerade im Vorbelasteten Raum - also jener Raum, der laut derzeitiger Gesetzgebung den zu bevorzugenden Standort darstellt - PV-Freiflächenanlagen die am wenigsten raumverträgliche Lösung sind. Speziell die unzureichenden Ausprägungen der ökonomischen Eigenschaften - Gestehungskosten, Regelbarkeit und Volllaststunden - sind der Grund, warum die Photovoltaik hier nicht raumverträglich ist. Tat- 


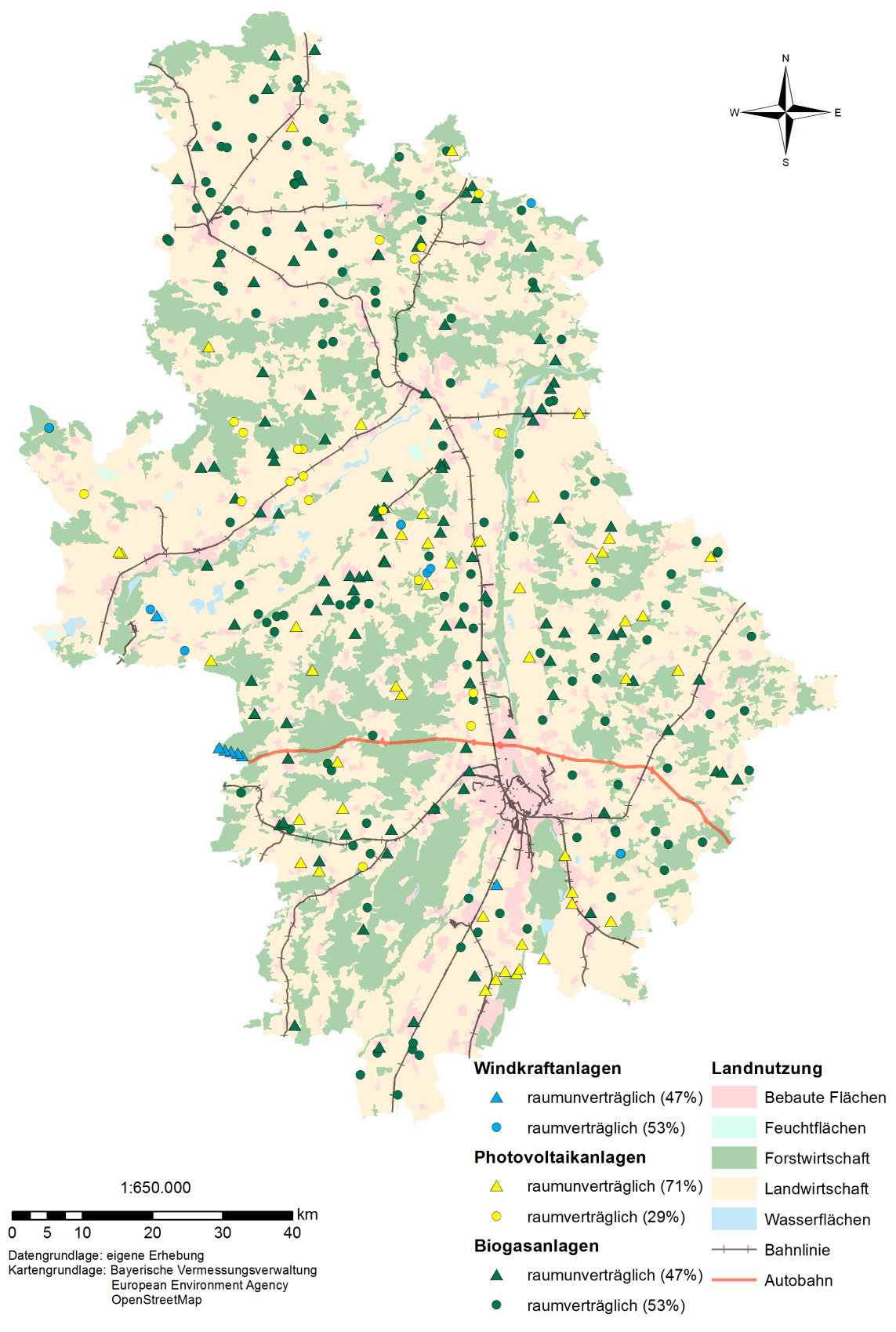

Abb. 7. Raumverträglichkeit der bisherigen Standortakquise.

sächlich gibt es erhebliche Probleme mit der Regelbarkeit und den Volllaststunden von PV-Anlagen, die jedoch entsprechend Schierenbeck et al. (2010) ein flächendeckendes Phänomen darstellen und schwer auf einen kleineren Raumausschnitt zu beziehen sind. Es muss auch nicht zwangsläufig so sein, dass es Probleme mit der Photovoltaik innerhalb dieser nicht zugeordneten Raumkategorie gibt, denn die Rangfolge der Technologien besagt ja letztlich nur, dass es eine Energieform gibt, - in diesem Fall die Biogasanlage -, die das Potenzial des Vorbelasteten Raumes besser erschließt.
Beim Forstwirtschaftlichen Raum kann die Photovoltaik, neben den drei genannten Parametern, zudem nicht den Raumanforderungen im Bereich Flächeneffizienz und Ökobilanz entsprechen. Alle anderen Technologien stellen bessere energetische Raumnutzungen dar. Des Weiteren befinden sich mehr als die Hälfte der Biogasanlagen in der Planungsregion 9 auf Standorten, die nach den theoretischkonzeptionellen Überlegungen als geeignet erscheinen. Jene Biogasanlagen jedoch, die sich im Bereich des Dienstleistungsraumes befinden, stellen keine raumverträgliche Lö- 
sung dar. Hierfür entscheidend sind die nicht ausreichenden Ausprägungen der Standortparameter Flächeneffizienz, Genehmigung, Ökobilanz und Rückbaufähigkeit.

Die Anforderungen des Dienstleistungsraumes sind diesbezüglich für diesen Technologiepfad zu hoch bzw. Windenergie und Photovoltaik stellen raumverträglichere Lösungen dar. Interessant ist, dass es innerhalb dieser Teilräume tatsächlich zu großer Kritik am Ausbau von Biogasanlagen gekommen ist. In der Literatur spielt dabei immer wieder der bei Biogasanlagen schlecht ausgeprägte Standortparameter „Ökobilanz“ eine zentrale Rolle. Dabei geht es um Aspekte wie dem Umbruch von ökologisch hochwertigem Grünland (Bosch, 2008; Laggner et al., 2014) sowie um die Ausweitung des Anbaus von Silomais und der damit einhergehenden Verstärkung von Bodenerosion, Eutrophierung und Fischsterben (Bosch, 2010).

An dieser Stelle liefert der theoretisch-konzeptionelle Ansatz folglich eine plausible Erklärung für die Unverträglichkeit der regionalen Standortakquise. Die Anzahl an Windkraftanlagen innerhalb der Planungsregion ist noch relativ gering und die bisherige Standortakquise als bedingt raumverträglich anzusehen. Speziell im Bereich des Verletzlichen Raumes stellt Photovoltaik die verträglichere Technologiewahl dar. Zur Tiefengeothermie liegen bislang keine Projekte vor.

\section{Abschließende Bewertung}

Die vergleichende Analyse hat ergeben, dass viele der Standortentscheidungen in der Planungsregion 9 nur bedingt raumverträglich sind, mancherorts sogar Technologiepfade betreten werden, die basierend auf den hier vorgestellten Kriterien die schlechteste Option darstellen. Diesbezüglich konnte beispielhaft aufgezeigt werden, dass zumindest teilweise eine Übereinstimmung zwischen den aus theoretisch-konzeptioneller Sicht hierfür verantwortlichen sowie den regional tatsächlich wahrnehmbar schlecht ausgeprägten Standortparametern besteht (vgl. Ökobilanz bei Biogasanlagen).

Darüber hinaus ist es gelungen, eine Annäherung an die theoretischen Grundlagen einer raumverträglichen Zuordnung von erneuerbaren Energien zu leisten und erstmalig im Rahmen eines ganzheitlichen Planungsansatzes umzusetzen. Des Weiteren bietet dieses standorttheoretische Konzept die Möglichkeit, Wettbewerbsmechanismen zwischen regenerativen Technologien zu simulieren und damit besser verstehen zu können. Es lässt sich ermitteln, welche Eigenschaft einer erneuerbaren Energie dazu führt, dass diese nicht attraktiv genug für einen bestimmten Teilraum ist. Unternehmen, die dieser Technologieroute zuzuordnen sind, könnten so den Fokus von FuE auf diese spezifische(n) Eigenschaft(en) richten und den Wettbewerbsnachteil um die Ressource Raum korrigieren. Umgekehrt könnten die Vertreter der führenden Technologien erkennen, welche technologischen Eigen- schaften ihren Wettbewerbsvorteil bedingen. Es würde ein konstruktiver Wettbewerb um die ökonomische, ökologische und soziale Verträglichkeit von erneuerbaren Energien entstehen und so zu einer größeren Akzeptanz der Energiewende führen.

Danksagung. Unser besonderer Dank gilt Herrn Benedikt Korf als Editor sowie den beiden anonymen Gutachter/innen der Geographica Helvetica für ihre konstruktiven Anmerkungen und hilfreichen Impulse.

Edited by: B. Korf

Reviewed by: two anonymous referees

\section{Literatur}

Aitken, M.: Why we still don't understand the social aspects of wind power: a critique of key assumptions within the literature, Energ. Policy, 38, 1834-1841, 2010a.

Aitken, M.: Wind power and community benefits: Challenges and opportunities, Energ. Policy, 38, 6066-6075, $2010 \mathrm{~b}$.

Ballestrem, F. v.: Standortwahl von Unternehmen und Industriestandortpolitik, in: Finanzwissenschaftliche Forschungsarbeiten, Herausgeber: Schmölders, G., 44, Berlin, 1974.

Bathelt, H. und Glückler, J.: Wirtschaftsgeographie - Ökonomische Beziehungen in räumlicher Perspektive, Stuttgart, 319 pp., 2002.

BDEW: Bundesverband der Energie- und Wasserwirtschaft: Kraftwerksplanung und aktuelle ökonomische Rahmenbedingungen für Kraftwerke in Deutschland, Berlin, 39 pp., 2013.

Becker, S., Gailing, L., und Naumann, M.: Neue Energielandschaften - Neue Akteurslandschaften, Eine Bestandsaufnahme in Brandenburg, Berlin, 67 pp., $2012 \mathrm{a}$.

Becker, S., Gailing, L., und Naumann, M.: Neue Akteurslandschaften der Energiewende. Aktuelle Entwicklungen in Brandenburg, Raumplanung, 162, 42-46, 2012 b.

Birkner, P.: „Systemintegration erneuerbarer Energiequellen“ Das neue Energiesystem und die besondere Rolle des urbanen Raums, UmweltWirtschaftsForum, 21, 225-231, 2013.

Bosch, S.: Kulturlandschaften und Erneuerbare Energien - Auswirkungen auf Natur und Landschaft: Ökonomische Bedeutung und ökologische Probleme der Biogasgewinnung, in: Verein Rieser Kulturtage e. V., Herausgeber: Rieser Kulturtage - Eine Landschaft stellt sich vor, Dokumentation Band XVII, 125-128, 2008.

Bosch, S.: Rieser Kulturlandschaft und Erneuerbare Energien. Energiepflanzenbau und Pflege der Kulturlandschaft - Zwischen Dichotomie und Synergie, in: Verein Rieser Kulturtage e. V., Herausgeber: Rieser Kulturtage - Eine Landschaft stellt sich vor, Dokumentation Band XVIII, 192-198, 2010.

Bosch, S.: Erfassung und Bewertung des Einflusses der Ressource Raum im Rahmen der Förderung von Erneuerbaren Energien sowie Ableitung eines ganzheitlichen Ansatzes zur optimalen Integration von regenerativen Technologien in den ländlichen Raum, Augsburg, 246 pp., 2012.

Bosch, S.: Geographie der erneuerbaren Energien - Räumliche Grenzen eines nachhaltigen Energiesystems, in: Die deutsche „Energiewende“ nach Fukushima. Der wissenschaftliche Diskurs zwischen Atomausstieg und Wachstumsdebatte, Herausge- 
ber: Radtke, J. und Hennig, B., „Beiträge zur sozialwissenschaftlichen Nachhaltigkeitsforschung“, 8, 397-422, Marburg, 2013.

Böttcher, J.: Finanzierung von Erneuerbare-Energien-Vorhaben, München, 277 pp., 2009.

Brücher, W.: Erneuerbare Energien in der globalen Versorgung aus historisch-geographischer Perspektive, Geographische Rundschau, 60, 4-12, 2008.

BMJV: Bundesministerium der Justiz und für Verbraucherschutz, Gesetz zum Schutz vor schädlichen Umwelteinwirkungen durch Luftverunreinigungen, Geräusche, Erschütterungen und ähnliche Vorgänge (BImSchG), verfügbar unter: http://www. gesetze-im-internet.de/bundesrecht/bimschg/gesamt.pdf (letzter Zugriff: 22.11.2015), Berlin, 2014.

BMVBS: Bundesministerium für Verkehr, Bau und Stadtentwicklung, Bundesinstitut für Bau-, Stadt- und Raumforschung (BBSR), und Bundesamt für Bauwesen und Raumordnung (BBR): Handlungskatalog: „Optionen erneuerbarer Energien im Stadtraum“, Bonn, 53 pp., 2008.

BMVBS: Bundesministerium für Verkehr, Bau und Stadtentwicklung, Bundesinstitut für Bau-, Stadt- und Raumforschung (BBSR), und Bundesamt für Bauwesen und Raumordnung (BBR): Nutzung städtischer Freiflächen für erneuerbare Energien, Bonn, 150 pp., 2009.

Czisch, G.: Szenarien zur zukünftigen Stromversorgung - Kostenoptimierte Variationen zur Versorgung Europas und seiner Nachbarn mit Strom aus erneuerbaren Energien, Kassel, 508 pp., 2005.

Daly, H. E.: Unwirtschaftliches Wachstum und Globalisierung in einer vollen Welt, Natur und Kultur, 2, 3-22, 2001.

Die Bundesregierung: Energiewende - Maßnahmen im Überblick, verfügbar unter: https://www.bundesregierung.de/Content/ DE/StatischeSeiten/Breg/Energiekonzept/0-Buehne/ma\%C3\% 9Fnahmen-im-ueberblick.html (letzter Zugriff: 12.01.2016), 2015.

Ecofys: Solare Flächenpotentiale Berlin: Stadtraumtypenkatalog, verfügbar unter: http://www.stadtentwicklung.berlin.de/ umwelt/umweltatlas/download/0806_Stadtraumtypen.pdf (letzter Zugriff: 15.11.2015), Berlin, 2008.

Einig, K., Heilmann, J., Zaspel, B.: Wie viel Platz die Windkraft braucht, neue energie, 21, 34-37, 2011.

Ellis, G., Barry, J., and Robinson, C.: Many ways to say „no“, different ways to say ,yes': applying Q-methodology to understand public acceptance of wind farm proposals, J. Environ. Plann. Man., 50, 517-551, 2007.

Gailing, L. und Röhring, A.: Was ist dezentral an der Energiewende? Infrastrukturen erneuerbarer Energien als Herausforderungen und Chancen für ländliche Räume, Raumforschung und Raumordnung, 73, 31-43, 2015.

Gailing, L., Hüesker, F., Kern, K., und Röhring, A.: Die räumliche Gestaltung der Energiewende zwischen Zentralität und Dezentralität. Explorative Anwendung einer Forschungsheuristik, Leibniz-Institut für Regionalentwicklung und Strukturplanung, 51, Erkner, 2013.

Heindl, P., Schüßler, R., und Löschel, A.: Ist die Energiewende sozial gerecht?, Wirtschaftsdienst, 94, 508-514, 2014.

Heinrichs, H.: Dezentral und partizipativ? Möglichkeiten und Grenzen von Bürgerbeteiligung zur Umsetzung der Energiewende, in: Die deutsche „Energiewende“ nach Fukushima. Der wissenschaftliche Diskurs zwischen Atomausstieg und Wachstumsde- batte, Herausgeber: Radtke, J. und Hennig, B., „Beiträge zur sozialwissenschaftlichen Nachhaltigkeitsforschung,,, Marburg, 8, 119-138, 2013.

Herbes, C. und Friege, C. (Hrsg.): Handbuch Finanzierung von Erneuerbare-Energie-Projekten, Konstanz und München, 456 pp., 2015.

IdE: Institut dezentrale Energietechnologien, 100ee-Regionen in Deutschland, Europa und der Welt, verfügbar unter: www.100-ee.de/downloads/broschueren/?eID=dam_frontend_ push\&docID=1396 (letzter Zugriff: 07.10.2015), 2014.

ISE: Fraunhofer-Institut für solare Energiesysteme, Stromgestehungskosten erneuerbarer Energien, Freiburg, 48 pp., 2013.

Iwes: Fraunhofer Institut für Windenergie und Energiesystemtechnik, Studie zum Potenzial der Windenergienutzung an Land, 24 pp., 2011.

Jakubowski, P. und Koch, A.: Energiewende, Bürgerinvestitionen und regionale Entwicklung, Informationen zur Raumentwicklung, 9/10, 475-490, 2012.

Jedicke, E.: Windmühlen von heute - ein Zielkonflikt des Naturschutzes, Naturschutz und Landschaftsplanung, 29, 196, 1997.

Kallmünzer, B.: Photovoltaische Freiflächenanlagen - energiewirtschaftliche und planungsrechtliche Rahmenbedingungen und GIS-gestützte Standortsuche am Beispiel der Gemeinde Kirchweidach, Landkreis Altötting, in: Arbeitsmaterialien Wirtschaftsgeographie Regensburg, Herausgeber: Schmude, J. und Klein, K., 14, Regensburg, 2006.

Kaltschmitt, M., Streicher, W., und Wiese, A. (Hrsg.): Erneuerbare Energien - Systemtechnik, Wirtschaftlichkeit, Umweltaspekte, 4. Aufl., Berlin, 2006.

Kammer, J.: Die Windenergieindustrie. Evolution von Akteuren und Unternehmensstrukturen in einer Wachstumsindustrie mit räumlicher Perspektive, in: Mitteilungen der Geographischen Gesellschaft in Hamburg, Herausgeber: Nagel, F. N., 103, Hamburg, Stuttgart, 2011.

Kanning, H., Buhr, N., und Steinkraus, K.: Erneuerbare Energien Räumliche Dimensionen, neue Akteurslandschaften und planerische (Mit)Gestaltungspotenziale am Beispiel des Biogaspfades, Raumforschung und Raumordnung, 67, 142-156, 2009.

Keil, M., Bock, M., Esch, T., Metz, A., Nieland, S., und Pfitzner, A.: CORINE Land Cover Aktualisierung 2006 für Deutschland. Abschlussbericht zu den F+E Vorhaben UBA FKZ 370712200 und FKZ 370812 200, DLR, Oberpfaffenhofen, 2010.

Kirchhoff, T.: Energiewende und Landschaftsästhetik, Naturschutz und Landschaftsplanung, 46, 10-16, 2014.

Klagge, B. und Arbach, C. (Hrsg.): Governanceprozesse für erneuerbare Energien. Akademie für Raumforschung und Landesplanung (=ARL-Arbeitsbericht 5), verfügbar unter: http://shop. arl-net.de/media/direct/pdf/ab/ab_005/ab_005_gesamt.pdf (letzter Zugriff: 12.01.2016), Hannover, 2013.

Klagge, B. und Brocke, T.: Energiewende vor Ort: Dezentrale Stromerzeugung und die Rolle von Stadtwerken und Regionalversorgern, Geographische Rundschau, 65, 12-18, 2013.

Klandt, H.: Aktivität und Erfolg des Unternehmensgründers. Eine empirische Analyse unter Einbeziehung des mikrosozialen Umfeldes, Bergisch Gladbach, 450 pp., 1984.

Kopp, O., Eßer-Frey, A., und Engelhorn, T.: Können sich erneuerbare Energien langfristig auf wettbewerblich organisierten Strommärkten finanzieren?, Zeitschrift für Energiewirtschaft, 36, 243 255, 2012. 
Kühne, O.: Heimat und sozial nachhaltige Landschaftsentwicklung, Raumforschung und Raumordnung, 69, 291-301, 2011.

Kulke, E.: Wirtschaftsgeographie, 5. aktualisierte Aufl., Paderborn, 356 pp., 2013.

Kunze, C. und Becker, S.: Wege der Energiedemokratie. Emanzipatorische Energiewenden in Europa, Brüssel, 160 pp., 2015.

Laggner, B., Orthen, N., Osterburg, B., und Röder, N.: Ist die zunehmende Biogasproduktion die alleinige Ursache für den Grünlandschwund in Deutschland? - eine Analyse von georeferenzierten Daten zur Landnutzung, Raumforschung und Raumordnung, 72, 195-209, 2014.

Ludwig, N. und Bosch, S.: Standortalternativen für die Windenergie - Berechnung des Ertragspotenzials auf Offenland- und Waldstandorten in Bayern, Raumforschung und Raumordnung, 72, 293-308, 2014.

Megerle, H.: Landschaftsveränderungen durch Raumansprüche erneuerbarer Energien - aktuelle Entwicklungen und Forschungsperspektiven am Beispiel des ländlichen Raumes in BadenWürttemberg, in: Neue Energielandschaften - Neue Perspektiven der Landschaftsforschung, Herausgeber: Gailling, L. und Leibenath, M., 145-164, Wiesbaden, 2013.

Moss, T., Gailing, L., Kern, K., Naumann, M., und Röhring, A.: Energie als Gemeinschaftsgut? Anregungen für die raumwissenschaftliche Energieforschung, Leibniz-Institut für Regionalentwicklung und Strukturplanung, 50, Erkner, 2013.

Nohl, W.: Landschaftsästhetische Auswirkungen von Windkraftanlagen, in: Bayerischer Landesverein für Heimatpflege e. V. (Hrsg.), Schönere Heimat - Erbe und Auftrag, 99, 3-12, 2010.

Paschen, H., Oertel, D., und Grünwald, R.: Möglichkeiten geothermischer Stromerzeugung in Deutschland. Arbeitsbericht Nr. 84 Büro für Technikfolgenabschätzung beim Deutschen Bundestag, 2003.

Peters, J.: Erneuerbare Energien und Kulturlandschaft, verfügbar unter: http://www.raumplanung.tu-dortmund.de/ves/cms/de/ Forschung/MORO-Forschungsprojekt/Expertentagung2008/ Expertentagung2008_Tagungsbeitraege/Peters_EE-Landschaft_ .pdf, (letzter Zugriff: 15.02.2016), Dortmund, 2008.

Peters, J., Torkler, F., Hempp, S., und Hauswirth, M.: Ist das Landschaftsbild „,berechenbar“"? - Entwicklung einer GIS-gestützten Landschaftsbildanalyse für die Region Uckermark-Barnim als Grundlage für die Ausweisung von Windeignungsgebieten, Naturschutz und Landschaftsplanung, 41, 15-20, 2009.

Peyke, G., Bosch, S., Färber, K., Tatu, D., und Walter, K.: Erneuerbare Energien von A bis Z, in: Geographica Augustana, 13, Augsburg, 2013.

Pred, A. R.: Behavior and Location: Foundations for a Geographic and Dynamic Location Theory: Part 1., Lund, 1967.

Prinz, T., Biberacher, M., Gadocha, S., Mittlböck, M., Schardinger, I., Zocher, D., Riedler, W., Strasser, H., Fackler, A., Dorfinger, N., und Obersteiner, M.: Energie und Raumentwicklung. Räumliche Potenziale erneuerbarer Energieträger, in: Österreichische Raumordnungskonferenz (ÖROK) (Hrsg.), 178, Wien, 2009.

Porsche, L.: Stadtentwicklung voller erneuerbarer Energie, Informationen zur Raumentwicklung, 9, 665-673, 2010.

Schardinger, I., Botzenhart, F., Biberacher, M., Hamacher, T., and Blaschke, T.: Integrating spatial models into regional energy system optimisation: focusing on biomass, International Journal of Energy Sector Management, 6, 5-32, 2012.
Scharnitzky, R., Szymanski, M., und Sebald, C.: Kabinett zieht den Stecker, Süddeutsche Zeitung 29, vom 5. Februar 2014, 33, 2014.

Schätzl, L.: Wirtschaftsgeographie 1: Theorie. 8. Aufl., Paderborn, 2001.

Scheidler, A.: Verunstaltung des Landschaftsbildes durch Windkraftanlagen, Natur und Recht, 32, 525-530, 2010.

Schierenbeck, S., Graeber, D., Semmig, A., und Weber, A.: Ein distanzbasiertes Hochrechnungsverfahren für die Einspeisung aus Photovoltaik, Energiewirtschaftliche Tagesfragen, 60, 60-64, 2010.

Schmude, J.: Geförderte Unternehmensgründungen in BadenWürttemberg: Eine Analyse der regionalen Unterschiede des Existenzgründungsgeschehens am Beispiel des Eigenkapitalhilfe-Programms (1979 bis 1989), Stuttgart, 1994.

Schöbel, S.: Windenergie und Landschaftsästhetik. Zur landschaftsgerechten Anordnung von Windfarmen, Berlin, 158 pp., 2012.

Smith, D. M.: Industrial Location: An Economic Geographical Analysis, New York, 506 pp., 1971.

Starick, A., Klöckner, K., Möller, I., Gaasch, N., und Müller, K.: Entscheidungshilfen für eine nachhaltige räumliche Entwicklung der Bioenergiebereitstellung - Methoden und ihre instrumentelle Anwendung, Raumforschung und Raumordnung, 69, 367-382, 2011.

StMI, StMWFK, StMF, StMWIVT, StMUG, StMELF: Bayerische Staatsministerien, Hinweise zur Planung von Windkraftanlagen, 65pp., 2011.

StMWI: Bayerisches Staatsministerium für Wirtschaft und Medien, Energie und Technologie, Energie-Atlas Bayern 2.0 Mischpult „Energiemix Bayern vor Ort“, verfügbar unter: http:// www.energieatlas.bayern.de/kommunen/mischpult.html (letzter Zugriff: 17.01.2016), 2012.

Tobias, K., Jung, C., Fallen, M., und Puga, J.-F.: Hinweise zur Planung von Windenergieanlagen auf Waldstandorten, Kaiserslautern, 53 pp., 2003.

Weiss, G.: Das Klima retten - aber nicht vor der eigenen Tür? Konflikte um Anlagen zur regenerativen Energieerzeugung in Deutschland, Geographische Rundschau, 65, 44-49, 2013.

Wenzel, B., Ohlhorst, D., und Bruns, A.: Geothermische Stromerzeugung in Deutschland - Stiefkind oder schlafender Riese? Zeitschrift für Energiewirtschaft, 2, 23-30, 2009.

Zepf, V., Reller, A., Rennie, C., Ashfield, M., and Simmons, J.: Materials critical to the energy industry. An introduction. 2nd edition, London, 2014.

Zimmerer, K.: Approaching the New Geographies of Global Energy: Analytics and Assessment of Current Energy Landscapes and Alternatives, in: The New Geographies of Energy. Assessment and Analysis of Critical Landscapes, Herausgeber: Zimmerer, K., 1-10, London, New York, 2013.

Zink R.: Einbindung von GIS in einen nachhaltigen Planungsprozess von Photovoltaik-Freiflächenanlagen auf kommunaler Ebene, in: Angewandte Geoinformatik 2010 - Beiträge zum 22. AGIT-Symposium Salzburg, Herausgeber: Strobl, J., Blaschke, T., und Griesebner, G., 954-963, 2010. 